The IJA is a peer-reviewed open-access, electronic journal, freely available without charge to users

Produced by the AquacultureHub non-profit Foundation Sale of IJA papers is strictly forbidden

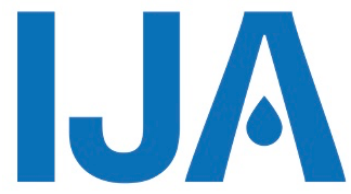

\title{
Dietary resveratrol attenuates oxidative stress in Pacific whiteleg shrimp, Litopenaeus vannamei, in response to ammonia stress
}

\author{
Huiling Liu, Zheng Yang, Yongxiong Huang, Shiping Yang*, \\ Yucong Huang, Shuanghu Cai, Jichang Jian
}

Fisheries College of Guangdong Ocean University, Southern Marine Science and Engineering Guangdong Laboratory (Zhanjiang), Guangdong Provincial Key Laboratory of Pathogenic Biology and Epidemiology for Aquatic Economic Animals \& Key Laboratory of

Control for Diseases of Aquatic Economic Animals of Guangdong Higher Education Institutes, Zhanjiang, China

Keywords: Litopenaeus vannamei; Resveratrol; Ammonia; Oxidative stress; protective effect

\begin{abstract}
To study the effect of resveratrol on the antioxidant system of Litopenaeus vannamei, resveratrol $(0,40,80,120$ and $180 \mathrm{mg} / \mathrm{kg})$ was added to the feed. After 7 days of culture, 10 tails of $L$. vannamei were randomly selected and exposure to ammonia stress at the sublethal concentration of ammonia $(20 \mathrm{mg} / \mathrm{L})$ for $24 \mathrm{~h}$. The results showed that the malondialdehyde (MDA) level in serum of shrimp fed with resveratrol decreased significantly compare to the control group $(p<0.05)$. After the ammonia stress, the MDA levels in serum in the Res 120 group and the Res 160 group was lower significantly than that in the control group $(p<0.05)$. The activities of superoxide dismutase (SOD) in the hepatopancreas and the serum, and glutathione peroxidase (GPX) in the serum of shrimps fed with resveratrol showed a decrease trend before the ammonia stress. Conversely, the activities of SOD and the total antioxidant competence (T-AOC) levels in the hepatopancreas in the Res 80 group and Res 120 group were significantly higher than that in the control group after the ammonia stress $(p<0.05)$. Dietary resveratrol decreased oxidative damage, increased antioxidant enzyme activities under the ammonia stress. Our results demonstrated that resveratrol attenuated oxidative stress and improved the resistance to the ammonia stress.
\end{abstract}

*Corresponding author: Fisheries College of Guangdong Ocean University, Zhanjiang, Guangdong, China, 524088. E-mail: ysp20010@sina.com 


\section{Introduction}

Shrimp culture has been become an important part of aquaculture, and the Pacific whiteleg shrimp, Litopenaeus vannamei, is the most important farmed shrimp specie worldwide with a total of 1.82 million tons production in China in 2019 (Ministry of Agriculture and Rural Affairs of PRC, 2020). However, with the expansion of demand for shrimp production, the cultivation scale and the cultivation density are increasing. Shrimp subjects to serious environmental stress, such as ammonia nitrogen, nitrite and unnormal $\mathrm{pH}$, which could increase oxidative stress and the susceptibility of shrimp to pathogens, and lead to the outbreak and epidemic of diseases (Tseng et al., 2004; Li et al., 2008; Liu et al., 2015a; Liang et al., 2016; Wang et al., 2020a). Ammonia nitrogen (ammonia), a common environmental stressor for aquatic animals, originates from excretion of the animals and decomposing organic wastes such as residual feed and feces in the water. Ammonia exposure induced oxidative stress, endoplasmic reticulum stress and apoptosis in hepatopancreas, inhibited the expressing of some immune response genes, and increased the malonyldialdehyde (MDA) level (Liang et al., 2016; Lu et al., 2016). Furthermore, ammonia could inhibit growth and resistance to pathogens, cause mortality of shrimp (Liu et al., 2004; Rostami et al., 2019).

Shrimp, just like any other organism, has a strong antioxidant system including antioxidant enzymes such as superoxide dismutase (SOD), peroxidase (POD), catalase (CAT), glutathione peroxidase (GPX) and some non-enzymatic antioxidants such as reduced glutathione, metallothionein, ascorbic acid, caratenoids. The antioxidant system maintains a balance of the generation and clearance of reactive oxygen species (ROS) in organism (Xu et al., 2012; Chen et al., 2020; Ebadi et al., 2020; Yu et al., 2020). Supplemental exogenous antioxidant can increase the antioxidant capacity. Therefore, some preventive and remedial additive agents were studied and applied in shrimp farming (Chien et al., 2003; Kitikiew et al., 2015; Shan et al., 2019; Chen et al., 2020). Resveratrol (trans-3,4,5-Trihydroxystilbene) is a natural polyphenol found in particularly high concentrations in red wine as well as numerous plants, such as grapes, peanuts, and other groundnuts. It has been attracted intense interest for its potentially beneficial effects and the mechanism of action (Robb, et al., 2008; Wang et al., 2019). It exhibits anti-oxidant, anti-inflammatory, anti-aging and anti-tumorigenic effects (Robb et al., 2008; Kim et al., 2018; Wang et al., 2019). Previous studies have reported that resveratrol could promote growth (Wilson et al., 2015), attenuate the oxidative damage (Liu et al., 2015b), increase antioxidant capability (Wang et al., 2020b), improve lipid and glucose metabolism (Zhang et al., 2017), protect from liver injury, inflammation and immunotoxicity induced by $\mathrm{H}_{2} \mathrm{O}_{2}$ (Jia et al., 2019) in aquaculture. However, whether resveratrol affects the oxidative status of shrimp has not yet been reported. Thus, we evaluated the effects of resveratrol on oxidative stress and resistance to ammonia by investigating oxidant stress status and antioxidant capacity with the aim of understanding the mechanism of action.

\section{Experimental animal and Diets}

\section{Materials and methods}

The study was performed using a batch of apparently healthy shrimps ( $L$. vannamei) from a shrimp farm at Donghai Island, Zhanjiang, Guangdong province. The average body weights of the shrimp were $11.09 \pm 0.51 \mathrm{~g}$. Shrimps were acclimated to experimental conditions in an indoor water system for $7 \mathrm{~d}$ before the experiment began. In this study, Yuehai brand commercial shrimp diet was used as the fundamental diet. The diet contains $40 \%$ of crude protein, $4.0 \%$ of crude fat, $5.0 \%$ of crude fibre, $16.0 \%$ of ash and $12 \%$ of moisture. Resveratrol (98\%) was bought from Harveybio (Beijing) Gene Technology Co., Ltd. Five different diets containing different resveratrol levels $(0,40,80$, 120 and $160 \mathrm{mg} \mathrm{kg-1}$, as Diet 1, Diet 2, Diet 3, Diet4 and Diet 5) were prepared based on the commercial diet. The feed was covered with $1 \%$ feed adhesive (Hainan Zhuoyue Biology Co., Ltd). Diet without resveratrol was used as control diet.

\section{Experimental design}

The Israeli Journal of Aquaculture - Bamidgeh • IJA.73.2021.1401912 
The experiment was carried out in an indoor rearing system. The experiment was divided into two phases: a $7 \mathrm{~d}$ feeding trial and a $24 \mathrm{~h}$ ammonia stress trial. At the beginning of the feeding trial, 450 healthy shrimps were randomly assigned into 15 plastic tanks (300 L) with $200 \mathrm{~L}$ water at a density of 30 shrimps per tank. Each treatment has three replicates. The shrimps in control group, Res 40 group, Res 80 group, Res 120 group and Res 160 Group were fed with Diet 1, Diet 2, Diet 3, Diet 4 and Diet 5, respectively. Shrimps was fed daily at 6:00A.M., 14:00 P.M., and 20:00 A.M. at normal day-night illumination. Residual diets and faces were removed by siphoning, and then about 15$30 \%$ of the total water volume was renewed daily to supplement the water siphoned off. During the feeding trial, the water was aerated continuously; the $\mathrm{pH}$, salinity and temperature of the water in tanks during the trial were $8.1 \pm 0.1,28.0 \mathrm{ppt}$ and $28.0^{\circ} \mathrm{C}$, respectively. The natural filtered seawater was used.

After the feeding trial, ammonia stress trial was performed. At the end of the feeding trial, 10 shrimps in the intermoult per tank were collected for the ammonia stress trial. Shrimps exposed to the sublethal concentration of ammonia ( $20 \mathrm{mg} / \mathrm{L}$ total ammonia) for 24h. $\mathrm{NH}_{4} \mathrm{Cl}$ was added to adjust to the ammonia concentration. During ammonia stress trial, the water wasn't aerated and renewed to ensure the stability of the ammonia concentration and minimize environmental impacts.

\section{Sample collection}

All animal sampling and handling procedures were followed in accordance with the standard guidelines and regulations on the use of animals for scientific research and purpose. To estimate oxidative stress, 5 individuals were sacrificed on ice for haemolymph and tissue collection for the analysis of antioxidant parameters at the end of the feeding trial and the ammonia stress trial, respectively. Haemolymph samples were rapidly collected from the sternal sinus located at the ventral part of cephlathorax with a syringe, and transferred to a sterile microcentrifuge tube, then kept at $4^{\circ} \mathrm{C}$ for 12 $\mathrm{h}$. The serum was collected by centrifuging at $3000 \mathrm{~g}$ for $15 \mathrm{~min}$ at $4^{\circ} \mathrm{C}$, and then stored at $-80^{\circ} \mathrm{C}$. The excised hepatopancreas tissues were homogenized in Tris- $\mathrm{HCl}$ buffer $(\mathrm{pH}$ 7.4 ) at $4^{\circ} \mathrm{C}$. The supernatant fluids were collected after centrifuging at $4000 \mathrm{~g}$ for $10 \mathrm{~min}$ at $4^{\circ} \mathrm{C}$ for antioxidant parameters analysis.

\section{Analytical methods}

The levels of MDA and T-AOC, the activity of SOD, CAT and GPX were monitored by measuring the optical density (OD) value at $523 \mathrm{~nm}, 520 \mathrm{~nm}, 550 \mathrm{~nm}, 405 \mathrm{~nm}$ and $412 \mathrm{~nm}$, respectively, using commercial kits (Nanjing Jiancheng Bioengineering Institute, China) according to the instructions of the manufacturers.

\section{Statistical analysis}

The data were subjected to one-way analysis of variance (ANOVA) and Duncan's multiple range tests using the SPSS 21 statistical software (SPSS Inc., USA) and EXCEL 2016 (Microsoft, USA). With respect to the results of Duncan's multiple range test, different lowercase letters indicate significant differences between the different treatments groups at each sampling point.

\section{MDA concentration in different tissue}

\section{Result}

The MDA levels were investigated in the hepatopancreas and serum of shrimps at the end of the feeding trial and ammonia stress trial (Figure 1). Prior to the ammonia stress trial, the MDA Levels in the hepatopancreas of shrimps fed with resveratrol had decreased by $5.2 \%, 7.1 \%, 17.1 \%, 19.0 \%$, but no significant differences were observed. The MDA levels in the serum of shrimps fed with resveratrol diet were significantly lower than that in the control group ( $p<0.05$ ). The MDA level of shrimps fed with diet 4 containing $120 \mathrm{mg} \mathrm{kg}^{-1}$ resveratrol had decreased significantly by $57.45 \%$ (Figure 1A).

As shown in Figure 1B, the MDA levels in the serum in the Res 120 group and the Res 160 group after ammonia stress were significantly lower than that in the control group ( $p$ 
$<0.05)$. Surprised, the MDA levels in the hepatopancreas in the Res 120 group was higher than that in the control group $(p<0.05)$, while those in the other groups were lower than that in the control group. After ammonia stress, the MDA levels in the hepatopancreas show an increase trend compare to those before ammonia stress trial, conversely, those in the serum show a decrease trend.

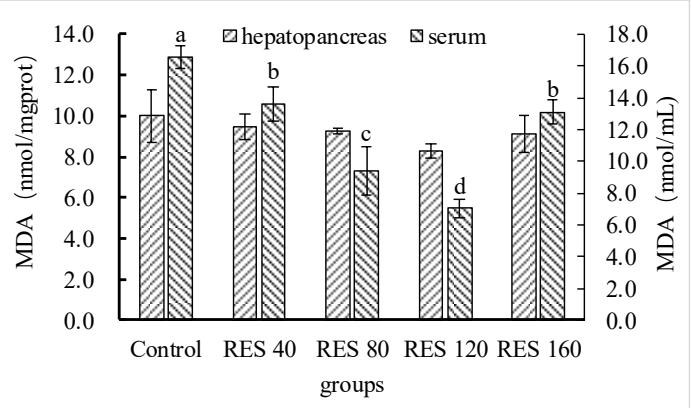

A

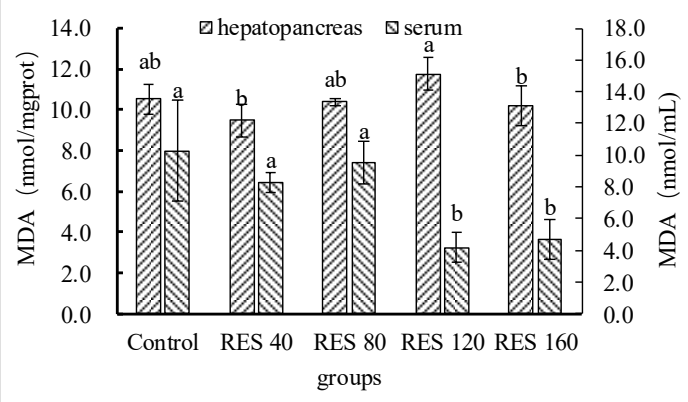

B

Figure 1 Effect of resveratrol on the MDA levels in different tissues of $L$. vannamei before (A) and after (B) the ammonia-N stress.

Notes: Different lower-case letters indicate significant differences between groups (the same as below). The primary $Y$ axis is used for the MDA levels in the hepatopancreas. The second $Y$ axis is used for the MDA levels in serum.

\section{Activity of SOD in different tissue}

As showed in Figure 2A, the activities of SOD in the hepatopancreas of shrimps fed with resveratrol diets were significantly lower than that in the control group $(p<0.05)$. The activities of SOD in the serum of shrimps fed with $120 \mathrm{mg} \mathrm{kg}^{-1}$ resveratrol diet was also significantly lower than that in the control group $(p<0.05)$.

After ammonia stress, the activities of SOD in the hepatopancreas of shrimps fed with resveratrol diets show an increase trend compare to that before ammonia stress trial (Figure 2B). The activities of SOD in the hepatopancreas in the Res 40 group, Res 80 group and Res 120 group were significantly higher than that in the control group $(p<0.05)$. There were no significant differences in the activities of SOD in the serum between all groups.
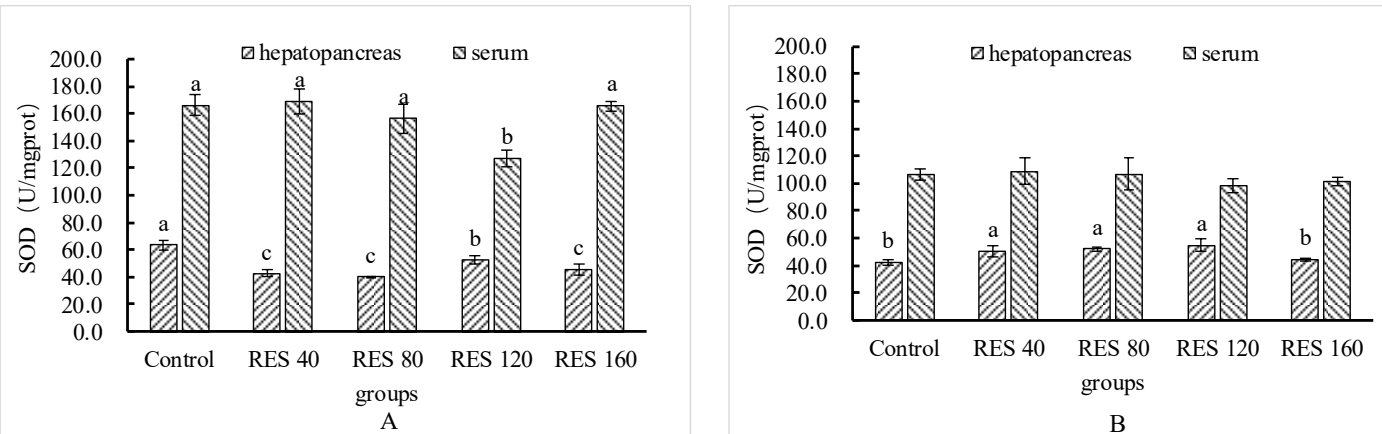

Figure 2 Effect of resveratrol on the activities of SOD in different tissues of $L$. vannamei before (A) and after (B) the ammonia-N stress.

\section{Activity of GPX in different tissue}

The activity of GPX of in the hepatopancreas and serum of the shrimps at the end of the feeding trial and ammonia stress trial were shown in Figure 3. The activities of GPX in all tissues of shrimps were affected significantly by resveratrol $(p<0.05)$. The activities of GPX in the hepatopancreas in the Res 40 group and Res 80 group were significantly lower than that in the control group $(p<0.05)$. Meantime, the activities of GPX in the serum in the Res 40 group, Res 120 group and Rev 120 group were significantly lower than that in the control group $(p<0.05)$ (Figure 3A). 
As showed in Figure 3B, the activities of GPX in the serum of the shrimps showed the same change trend compare to that before the ammonia stress. The activities of GPX in the serum in the control group and the Rev 80 group were higher than those in the Res 40 group and Res 120 group $(p<0.05)$. Compare to that before the ammonia stress trial, the activities of GPX in the hepatopancreas and serum of shrimps in all group showed a decreased trend.

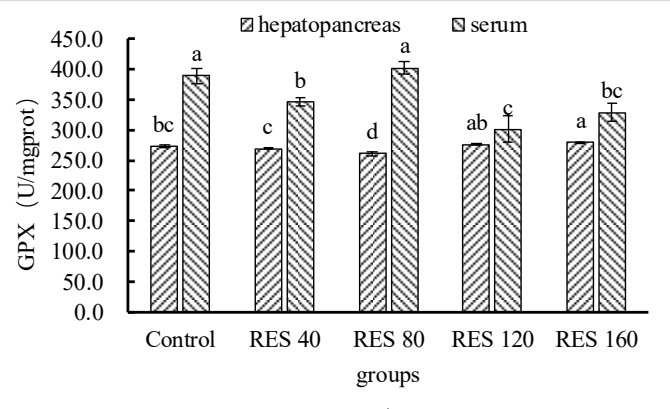

A

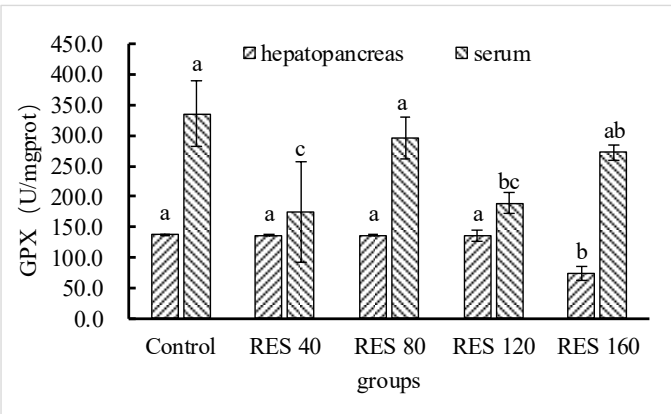

B

Figure 3 Effect of resveratrol on the activities of GPX in different tissues of L. vannamei before $(A)$ and after $(B)$ the ammonia- $N$ stress.

\section{Activity of CAT in different tissue}

The activities of CAT in the hepatopancreas and serum of shrimps were shown in Figure 4. The activities of CAT in the serum in the Res 120 group was significantly higher than that in the control group $(p<0.05)$. Although large difference in the activities of CAT in the hepatopancreas and serum were found, but no significantly differences were observed after the ammonia stress ( $p>0.05)$.
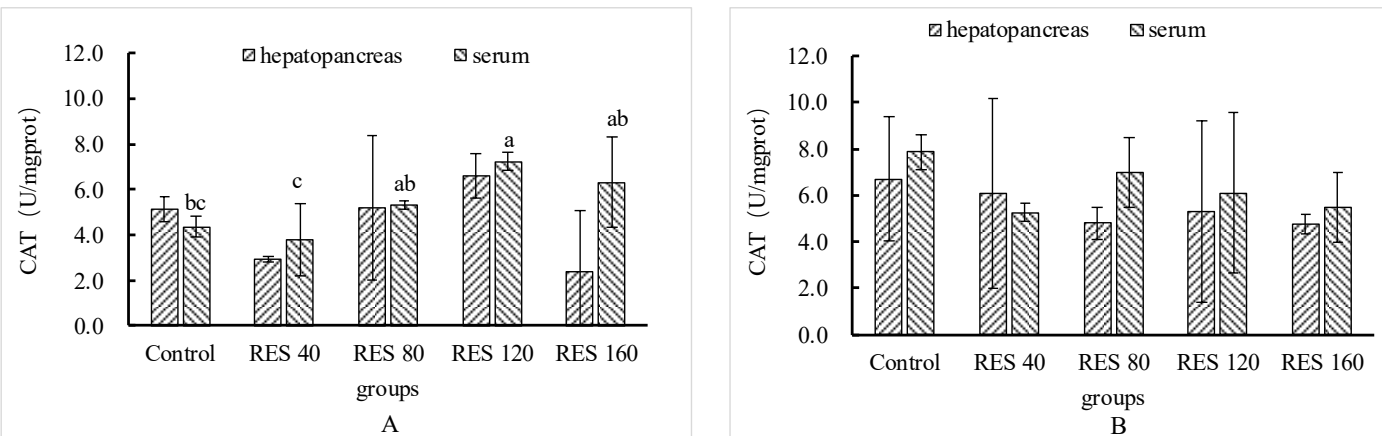

Figure 4 Effect of resveratrol on the activities of CAT in different tissues of $L$. vannamei before $(A)$ and after $(B)$ the ammonia-N stress.

\section{$T$-AOC level in different tissue}

The T-AOC levels in all tissues of shrimps were affected significantly by dietary resveratrol before the ammonia stress trial $(p<0.05)$ (Figure $\mathbf{5 A})$. The T-AOC levels in the hepatopancreas of the shrimps fed with resveratrol were significantly lower than that in the control group before the ammonia stress $(p<0.05)$. However, the T-AOC levels in the serum in the Res 120 group were significantly higher than that in the control group $(p<0.05)$. After the ammonia stress, the T-AOC levels in the hepatopancreas in the Res 80 group, Res 120 group and Res 160 group were significantly higher than that in the control group $(p<0.05)$. There is no significant difference in the serum between all groups after the ammonia stress. 


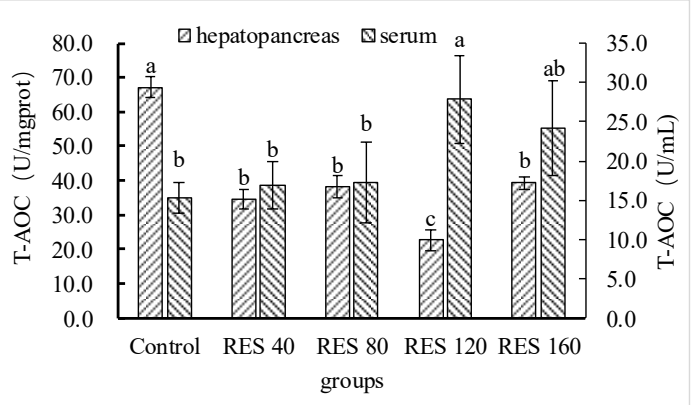

A

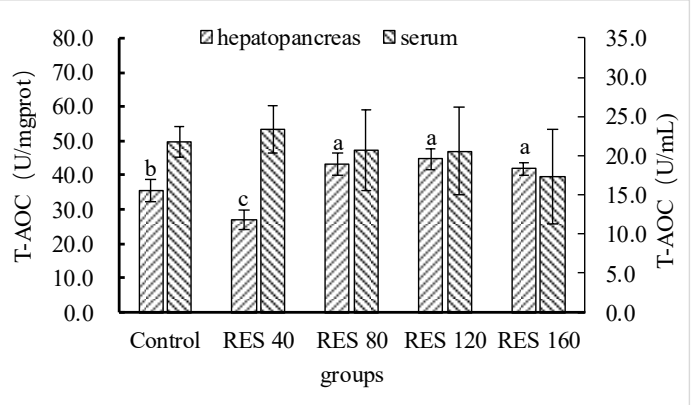

B

Figure 5 Effect of resveratrol on the T-AOC levels in different tissues of $L$. vannamei before (A) and after (B) the ammonia-N stress.

Notes: The primary $Y$ axis is used for the T-AOC levels in the hepatopancreas. The second $Y$ axis is used for T-AOC levels in serum.

\section{Discussion}

Effects of dietary resveratrol supplementation on MDA

Resveratrol is a naturally occurring antioxidant, and has been proved to have antioxidant effects in mammals. Nevertheless, there is a few reports about its effect on aquatic animals, especially shrimp. Prior studies have examined the effects of some remedial addition agents, such as probiotics and prebiotics (Kitikiew et al., 2015; Chen et al., 2020), feed organisms (Ampithoe sp.) (Shan et al., 2019), astaxanthin (Chien et al., 2003; Yu et al., 2020), vitamin C and vitamin E (Ebadi et al., 2020) on oxidative stress and antioxidant capacity in shrimp. Various parameters, MDA and protein carbonyl, are used as the biomarkers of oxidative damage. The MDA is one of the final products of lipid peroxidation, and is positively correlated with oxidative stress and lipid peroxidation. Astaxanthin contributed to dose-dependent protection against the oxidative damage induced by oxidized fish oil in L. vannamei (Yu et al., 2020). In this study, the MDA levels in the hepatopancreas and serum decreased compare to that in the control group. Moreover, the MDA level in the hepatopancreas in shrimp fed with resveratrol were significantly lower than that in the control groups before ammonia stress $(p<0.05)$. Briefly, the higher MDA levels revealed that the shrimps suffered more oxidative stress. These results demonstrated that resveratrol decreased the degree of the environmentalinduced increase of lipid peroxidation and the oxidative stress in shrimp. Our results are in accordance with other findings. Resveratrol treatment reduced significantly the increase of ROS and oxidative damage with age in a short-lived fish (Nothobranchius guentheri) (Liu et al., 2015b). Resveratrol also enhanced growth, increased antioxidant capacity, and limited protein and lipid damage in Paralichthys lethostigma (Wilson et al., 2015).

Hepatopancreas is as the metabolic center for reactive oxygen species production in crustacean, and is more sensitive to ROS (Han et al., 2018). After the ammonia stress, an increase trend of the MDA levels in the hepatopancreas in all groups was observed. Liang et al. reported that ammonia exposure could induce oxidative stress, and cause increase of the MDA level in L. vannamei (Liang et al., 2016). Dietary antioxidant, such as glutathione, reduced dose-dependently the MDA level in $L$. vannamei by the glutathione supplement antioxidant (Xu et al., 2012). In this study, the MDA levels in the serum in the Res 120 group and the Res 160 group were significantly lower than that in the control group after the ammonia stress $(p<0.05)$. This result indicated that pretreatment with resveratrol attenuated the oxidative damage by ammonia stress in $L$. vannamei. Which was consistent with the study that resveratrol suppressed the elevation of MDA in serum and liver in Oreochromis niloticus (Jia et al., 2019).

Effects of dietary resveratrol supplementation on antioxidant capacity

Under normal conditions, antioxidant system maintain a balance of the generation and clearance of reactive oxygen species in organism. However, cultured shrimp often suffers various environmental stresses, such as stocking density, physicochemical parameters (e.g., ammonia, nitrite, unnormal temperature and unnormal $\mathrm{pH}$ ) and pathogens. 
Organisms have a certain capacity to adapt these stresses, but excess stress affect the antioxidant capacity and the immune capacity (Marcadenti et al., 2015). Various antioxidant enzymes play an important role in their environmental adaption and antioxidant defense. Endogenous antioxidant enzymes, such as SOD, GPX and CAT, are the important and major enzymes used to balance out free radicals, eliminate these potentially hazardous and prevent from oxidative damage (Ifeanyi, 2018). The nuclear erythroid 2-related factor 2 (Nrf2) is a critical transcription factor, which activates the expression of the genes containing the antioxidant response element (ARE), and increase the activities of antioxidant enzymes (Dong et al., 2008). Resveratrol is an Nrf2 activator and an effective antioxidant that scavenges ROS and modulates physical performance preventing oxidative stress (Salomão et al., 2019; Farkhondeh et al., 2020). Resveratrol decreased the levels of ROS, elevated the activities of CAT, SOD and GPX, and exerted resveratrol increased protective effects on activities of antioxidant enzymes in $N$. guentheri (Liu et al, 2015). Dietary resveratrol upregulated the mRNA expressions of $\mathrm{Cu} / \mathrm{Zn}-\mathrm{SOD}, \mathrm{CAT}$ and GPx, and improve immunity, antioxidant capability and lipid metabolism of blunt snout bream (Megalobrama amblycephala) (Jia et al., 2019). Resveratrol also enhanced significantly the mRNA expression of SOD, GPX and the activity of SOD in turbot (Tan et al., 2019). Conversely, in this study, the activities of SOD and GPX, and the T-AOC levels in the hepatopancreas and serum of shrimps fed with resveratrol showed decreased trends before ammonia stress compare to that in the control group. The activities of SOD and the T-AOC levels in the hepatopancreas of shrimps fed with resveratrol diets were significantly lower than that in the control group $(p<0.05)$. Considering the lower MDA levels, we speculated that the decreased enzyme activity of SOD and GPX of shrimps fed with resveratrol might be an adaptive response to lower oxidative stress. Fish (Hyphessobrycon callistus) fed with carotenoid had lower SOD and GPX (Wang et al., 2006). Dietary astaxanthin also reduced the SOD activity in Tiger prawn (Penaeus monodon) (Chien et al., 2003). The activities of SOD and CAT, and the MDA levels of Clarias gariepinus in clean region from Orontes river were higher than those in the polluted regions (Turan et al., 2020). The SOD activity of L. vannamei injected Vibro parahaemolyticus at $3 \mathrm{~h}, 6 \mathrm{~h}$ and $12 \mathrm{~h}$ were higher than that in the control group (Pang et al., 2019). These results indicated that organism with lower oxidative stress had a reduced need for endogenous antioxidant enzymes.

As mentioned above, hepatopancreas is more sensitive to ROS. In this study, an increase trend in the activities of SOD in the hepatopancreas of shrimps fed with resveratrol diets was observed compare to that before ammonia stress trial. The T-AOC levels in the hepatopancreas in the Res 80 group, Res 120 group and Res 160 group were also higher than that before ammonia stress. The increased activity of SOD and the T-AOC level of shrimp fed with resveratrol might be an adaptive response to the elevated oxidative stress, suggesting that the antioxidant capacity of shrimp was improved by dietary resveratrol. The expression of the SOD, and prophenoloxidase genes in shrimp fed with hydrolyzed yeast and/or Bucillus licheniformisup-regulated after ammonia challenge (Chen et al., 2020). Dietary astaxanthin improved the antioxidant capacity of $L$. vannamei by increasing the CAT activity (Yu et al., 2020). Consequently, the present research hypothesizes that resveratrol might play an important role in increase antioxidant capacity and decrease oxidative damage by environmental stress in shrimp.

\section{Acknowledgements}

This study was funded by the National Key R\&D Program of China (No. 2018YFD0900501), the Fund of Southern Marine Science and Engineering Guangdong Laboratory (Zhanjiang) (No. ZJW-2019-06). We are also grateful to all the people who have dedicated their time to the trials. 


\section{References}

Chen M., Chen X.Q., Tian L.X., Liu Y.J. and J. Niu, 2020. Enhanced intestinal health, immune responses and ammonia resistance in pacific white shrimp (Litopenaeus vannamei) fed dietary hydrolyzed yeast (Rhodotorula mucilaginosa) and Bacillus licheniformis. Aquaculture Reports, 17 , 10385. https://doi.org/10.1016/j.aqrep.2020.100385

Chien Y.H., Pan C.H., and B. Hunter, 2003. The resistance to physical stresses by Penaeus monodon juvenile fed diets supplemented with astaxanthin. Aquaculture, 216(14), 177-191. https://doi.org/10.1016/S0044-8486(02)00056-X

Dong J., Sulik K.K., and S.Y. Chen, 2008. Nrf2-mediated transcriptional induction of antioxidant response in mouse embryos exposed to ethanol in vivo: implications for the prevention of fetal alcohol spectrum disorders. Antioxidants \& Redox Signaling, 10(12), 2023-2033. https://doi.org/10.1089/ars.2007.2019

Ebadi H., Zakeri M., Mousavi S.M., Yavari V., and M. Souri, 2020. The interaction effects of dietary lipid, vitamin $E$ and vitamin $C$ on growth performance, feed utilization, muscle proximate composition and antioxidant enzyme activity of white leg shrimp (Litopenaeus vannamei) [J]. Aquaculture Research, 1-13. https://doi.org/10.1111/are.1505

Farkhondeh T., Folgado S.L., Pourbagher-Shahri A.M., Ashrafizadeh M., and S. Samarghandiane, 2020.The therapeutic effect of resveratrol: Focusing on the Nrf2 signaling pathway. Biomedicine \& Pharmacotherapy, 127, 110234. https://doi.org/10.1016/j.biopha.2020.110234

Han S.Y., Wang M.Q., Wang B.J., Liu M., Jiang K.Y., and L. Wang, 2018. A comparative study on oxidative stress response in the hepatopancreas and midgut of the white shrimp Litopenaeus vannamei under gradual changes to low or high $\mathrm{pH}$ environment. Fish \& Shellfish Immunology, 76, 27-34. https://doi.org/10.1016/j.fsi.2018.02.001

Ifeanyi O.E.,2018. A review on free radicals and antioxidants. International Journal of Current Research in Medical Sciences 4(2), 123-133. http://doi.org/10.22192/ijcrms.2018.04.02.019

Jia R., Li Y., Cao L., Du J., Zheng T., Qian H., Gu Z., Jeney G., Xu P., and G. Yin, 2019. Antioxidative, anti-inflammatory and hepatoprotective effects of resveratrol on oxidative stress-induced liver damage in tilapia (Oreochromis niloticus). Comparative Biochemistry and Physiology Part C: Toxicology \& Pharmacology, 2019, 215: 56-66. https://doi.org/10.1016/j.cbpc.2018.10.002

Kim E.N., Lim J.H., Kim M.Y., Ban T.H., and B.S. Choi, 2018. Resveratrol, an nrf2 activator, ameliorates aging-related progressive renal injury. Aging, 10(1), 83-99. https://doi.org/10.18632/agibg.101361

Li C.C., and J.C. Chen, 2008. The immune response of white shrimp Litopenaeus vannamei and its susceptibility to Vibrio alginolyticus under low and high pH stress. Fish \& Shellfish Immunology, 25(6), 701-709. https://doi.org/10.1016/j.fsi.2008.01.007

Liang Z., Liu Z., Wang L., and M. Sun, 2016.Ammonia exposure induces oxidative stress, endoplasmic reticulum stress and apoptosis in hepatopancreas of pacific white shrimp (Litopenaeus vannamei). Fish \& Shellfish Immunology, 2016, 54(1), 523-528. https://doi.org/10.1016/j.fsi.2016.05.00

Liu, C.H., and Chen, J.C., 2004.Effect of ammonia on the immune response of white shrimp Litopenaeus vannamei and its susceptibility to Vibrio alginolyticus. Fish \& Shellfish Immunology, 16(3), 321-334.https://doi.org/10.1016/S1050-4648(03)00113-X

Liu H.L., Yang S.P., Wang C.G., Chan S.M., and C.B. Sun, 2015a. Effect of air exposure and resubmersion on the behavior and oxidative stress of pacific white shrimp Litopenaeus vannamei. North American Journal of Aquaculture, 77(1), 43-49. https://doi.org/10.1080/15222055.2014.955157

Liu T., Qi H., Ma L., Liu Z., Fu H., Zhu W. Song T., Yang B. and G. Li, 2015. Resveratrol attenuates oxidative stress and extends life span in the annual fish Nothobranchius guentheri. Rejuvenation Reserch, 18(3), 225-233. https://doi.org/10.1089/rej.2014.1618 
Lu X., Kong J., Luan S., Dai P., Meng X., Cao B., and K. Luo, 2019.Transcriptome analysis of the hepatopancreas in the pacific white shrimp (Litopenaeus vannamei) under acute ammonia stress. Plos One, 11(10), e0164396. https://doi.org/10.1371/journal.pone.0164396

Marcadenti A., and R.C.L.A. Coelho,2015. Dietary antioxidant and oxidative stress: interaction between vitamins and genetics. Journal of Nutritional Health \& Food Science, 3(1), 1-7. https://doi.org/10.15226/jnhfs.2015.00138

Ministry of Agriculture and Rural Affairs P.R.C., 2020. China Fishery Statistical Yearbook. Beijing: China Agriculture Press, pp22-24 (In Chinese).

Kitikiew S., Chen Y.Y., Yeh S.T., and J.C. Chen, 2015. White shrimp Litopenaeus vannamei that have received fucoidan show protective immunity after ammonia stressing. Journal of the Fisheries Society of Taiwan, 42(3), 189-197. https://doi.org/10.13140/RG.2.2.25922.17602

Pang H., Wang G., Zhou S., Wang J., Zhao J,, Hoare R., Monaghan S.J., Wang Z., and C. Sun, 2019. Survival and immune response of white shrimp Litopenaeus vannamei following single and concurrent infections with WSSV and vibrio parahaemolyticus. Fish \& Shellfish Immunology, 92, 712-718. https://doi.org/10.1016/j.fsi.2019.06.039

Robb E.L., Page M.M., Wiens B.E., and J.A. Stuart, 2008. Molecular mechanisms of oxidative stress resistance induced by resveratrol: specific and progressive induction of MnSOD. Biochemical and Biophysical Research Communications, 367(2), 406-412. https://doi.org/10.1016/j.bbrc.2007.12.138

Rostami F., Davoodi R., Nafisi Bahabadi M., Salehi F., and H. Nooryazdan, 2019. Effects of ammonia on growth and molting of Litopenaeus vannamei post larvae reared under two salinity levels. Journal of Applied Aquaculture, 31(4): 1-13. https://doi.org/10.1080/10454438.2019.1593911

Salomão R.A.S., Paula T.G.G.D., Zanella B.T.T.Z., Carvalho P.L.P.F., Duran B.O.S., Valente J.S., Fantinatti, B.E.A., Fernandes A.A., Barros M.M., Mareco E.A., Carvalho R.F., Santos V.B., and M.D.P. Silva, 2019. The combination of resveratrol and exercise enhances muscle growth characteristics in pacu (Piaractus mesopotamicus). Comparative Biochemistry and Physiology Part A: Molecular a Integrative Physiology, 235, 46-55. https://doi.org/10.1016/j.cbpa.2019.05.002

Shan H., Wang T., Dong Y., and S. Ma, 2019. Effects of dietary Ampithoe sp. supplementation on the growth, energy status, antioxidant capacity, and ammonia- $\mathrm{N}$ tolerance of the shrimp Litopenaeus vannamei: continuous versus interval feeding. Aquaculture, 509, 32-39. https://doi.org/10.1016/j.aquaculture.2019.05.021

Tan C., Zhou H., Wang X., Mai K., and G. He, 2019. Resveratrol attenuates oxidative stress and inflammatory response in turbot fed with soybean meal based diet. Fish \& shellfish immunology, 91, 130-135. https://doi.org/10.1016/j.fsi.2019.05.030

Turan F., Eken M., Ozyilmaz G., Karan S., and H. Uluca, 2020. Heavy metal bioaccumulation, oxidative stress and genotoxicity in African catfish Clarias gariepinus from Orontes river. Ecotoxicology, 29(3). https://doi.org/10.1007/s10646-020-02253-w

Tseng, I.T., and J.C. Chen, 2004. The immune response of white shrimp Litopenaeus vannamei and its susceptibility to Vibrio alginolyticus under nitrite stress. Fish \& shellfish immunology,17(4), 325-333.https://doi.org/10.1016/S1050-4648(03)00113-X

Wang F., Liang Q., Liu C., Dong W., Ou M., Li Z., Liu Y., and W. Wang, 2020a. Tuberous sclerosis complex 1 (PVTSC1) participates in ammonia nitrogen induced oxidative stress in Penaeus vannamei by regulating autophagy. Aquaculture, 533, 736107. https://doi.org/10.1016/j.aquaculture.2020.736107

Wang G., Xie X., Yuan L., Qiu J., Duan W., Xu B., and X. Chen, 2019. Resveratrol ameliorates rheumatoid arthritis via activation of SIRT1 - Nrf2 signaling pathway. BioFactors, 46, 441-453. https://doi.org/10.1002/biof.1599

Wang M.C., Wang Y.C., Peng H.W., Hseu J.R., Wu G.C., Chang C.F., and Y.C. Tseng, 2020b. Resveratrol induces expression of metabolic and antioxidant machinery and protects tilapia under cold stress. International Journal of Molecular Sciences, 21(9), 3338. https://doi.org/doi:10.3390/ijms21093338 
Wang Y.J., Chien Y.H., and C.H. Pan, 2006. Effects of dietary supplementation of carotenoids on survival, growth, pigmentation, and antioxidant capacity of characins, Hyphessobrycon callistus. Aquaculture, 261(2), 641-648. https://doi.org/10.1016/j.aquaculture.2006.08.040

Wilson W.N., Baumgarner B.L., Watanabe W.O., Alam M.S., and S.T. Kinsey, 2015. Effects of resveratrol on growth and skeletal muscle physiology of juvenile southern flounder. Comparative Biochemistry and Physiology Part A: Molecular \& Integrative Physiology, 2015, 183, 27-35. https://doi.org/10.1016/j.cbpa.2014.12.014

Xu D.D., Liu X. H., Cao J.M., Du Z.Y., Huang Y.Y., Zhao H.X., and X. Zhu, 2012. Dietary glutathione as an antioxidant improves resistance to ammonia exposure in Litopenaeus vannamei. Aquaculture Research, 43, 311-316. https://doi.org/10.1111/j.1365-2109.2011.02820.x

Yu Y., Liu Y., Yin P., Zhou W., Tian L., Liu Y., Xu D., and J. Niu, 2020. Astaxanthin attenuates fish oil-related hepatotoxicity and oxidative insult in juvenile Pacific white shrimp (Litopenaeus vannamei). Marine Drugs, 18(4), 218. https://doi.org/10.3390/md18040218

Zhang D., Yan Y., Tian H., Jiang G., and W. Liu, 2017. Resveratrol supplementation improves lipid and glucose metabolism in high-fat diet-fed blunt snout bream. Fish Physiology \& Biochemistry, 44(1), 1-11. https://doi.org/10.1007/s10695-017-0421-9 